\title{
Robust Switching Control Strategy for a Transmission System with Unknown Backlash
}

\author{
Qiang Ling, ${ }^{1}$ Zhe Yan, ${ }^{1}$ Honghai Shen, ${ }^{2}$ Jiquan $\mathrm{Li}^{2}$, and Yongyang Wang ${ }^{2}$ \\ ${ }^{1}$ Department of Automation, University of Science and Technology of China, Hefei, Anhui 230027, China \\ ${ }^{2}$ Key Laboratory of Airborne Optical Imaging and Measurement of CAS, Changchun Institute of Optics, Fine Mechanics and Physics, \\ Chinese Academy of Sciences, Changchun 130033, China
}

Correspondence should be addressed to Qiang Ling; qling@ustc.edu.cn

Received 2 January 2014; Revised 20 March 2014; Accepted 20 March 2014; Published 7 April 2014

Academic Editor: Sebastian Anita

Copyright (C) 2014 Qiang Ling et al. This is an open access article distributed under the Creative Commons Attribution License, which permits unrestricted use, distribution, and reproduction in any medium, provided the original work is properly cited.

\begin{abstract}
This paper proposes a robust switching control strategy for a transmission system with unknown backlash. Firstly, the adverse effects of backlash nonlinearity in the transmission system are analyzed. Then the backlash model and different operating modes of the transmission system with backlash are investigated. For each operating mode, an individual controller is designed to make the system robust against the unknown backlash and various frequencies of the input signal. Moreover, a supervisory controller is proposed to estimate the current mode of the transmission system and coordinate the controller switching between different modes. Simulations are done to verify that our switching control strategy can efficiently reduce the oscillation caused by backlash and is quite robust against the variation of the frequency of the input signal.
\end{abstract}

\section{Introduction}

In industrial applications, the transmission structure with gears is widely used. This kind of transmission structure, however, usually leaves a gap between two contacting gear teeth, which stores lubricating oil and compensates the size change caused by the temperature and elastic deformation. In addition, some inevitable errors in the production and assembling of gears also produce a gap between the teeth of the gears which mesh each other.

In a transmission system with backlash, when the driving gear changes direction, the driven gear will be uncontrollable due to the existence of backlash. The driven gear cannot be controlled by the driving gear until the driving and driven gears contact again, which yields tracking error. Meanwhile, due to the existence of backlash, the relationship between the output shaft and the input shaft in the transmission system becomes nonlinear, which complicates the control task of such system. Moreover, when the driving gear and the driven gear contact again, there will be an impact which does harm to the tracking performance of the system. All the abovementioned factors will cause adverse effects on the control precision of the transmission system [1].
Some compensation methods have been proposed to mitigate the adverse effects caused by backlash in the transmission system. These methods can be classified into two types, compensation based on mechanical techniques and compensation based on control techniques.

Among mechanical-based methods, two-motor configuration was proposed in [2]. Although this method can eliminate the nonlinear effects of backlash, the cost of the twomotor configuration is high. And two-motor configuration will increase both the energy consumption and the overall weight of the system.

The control-based methods compensate the backlash nonlinearity through designing an appropriate control algorithm. In [3], a switching control strategy is implemented, which uses a proportional-integral controller for the mode of contact and a proportional controller for the mode of backlash. In this strategy, although the driving gear can pass the backlash smoothly and make a smaller impact when it contacts the driven gear again, the transient time is long when the frequency of input signal is low, which causes a poor transient performance. In the literature, the inverse model is widely used to handle backlash [4-6]. For the backlash model with unknown parameters, inverse model 


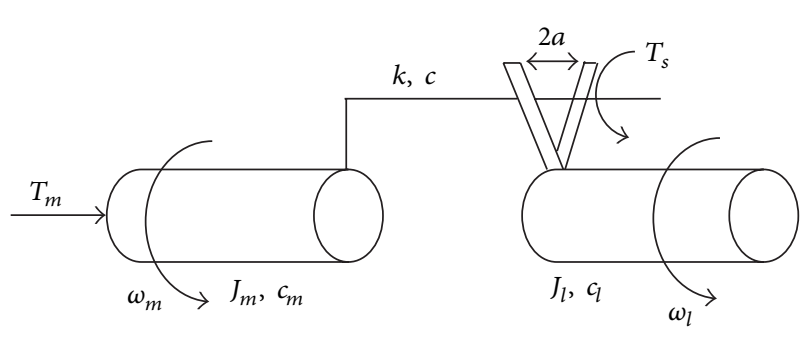

FIGURE 1: A schematic diagram of two-mass system with backlash.

can be built by estimating the unknown parameters online. Then the backlash nonlinearity can be eliminated by using the inverse model. However, the effectiveness of the inverse model largely depends on the accuracy of the backlash model. In practical applications, the size of backlash usually changes around the standard size due to the manufacturing precision and production environment, which makes the estimation error become inevitable. Because the accuracy of inverse model drops, we may not get satisfactory compensation performance.

In order to resolve the above issues, this paper proposes a robust switching control strategy for a transmission system with unknown backlash. In the contact mode, the system adopts a linear controller. In the backlash mode, the system adopts a composite controller which uses linear controller and backlash compensation controller together to eliminate the nonlinearity caused by unknown backlash. When designing the controller, we consider reducing not only the transient time but also the impact when gears contact again. Furthermore, the control strategy is robust against the frequency of the input signal. As simulations show, under this switching control strategy, the system has less steady-state error and oscillation, especially when the frequency of input signal is low.

The rest of this paper is organized as follows. In Section 2, we present the concerned backlash model. The details of our switching control strategy are given in Section 3. Simulation results are shown in Section 4. Some final remarks are placed in Section 5.

\section{Backlash Model}

In the survey paper [1], several backlash models have been mentioned, such as dead zone model, hysteresis model, and exact model. Particularly, the dead zone model is not only a good description of the backlash nonlinearity, but also relatively easy to implement. So, this paper adopts the dead zone model to describe the backlash.

Consider a two-mass system whose two parts are connected by gears. In order to simplify the analysis, assume that the gear ratio is 1 . If the gear ratio is not 1 , as long as the driven gear can be reduced to the driving gear, the subsequent equations still hold. A schematic diagram of the two-mass system with backlash is shown in Figure 1.

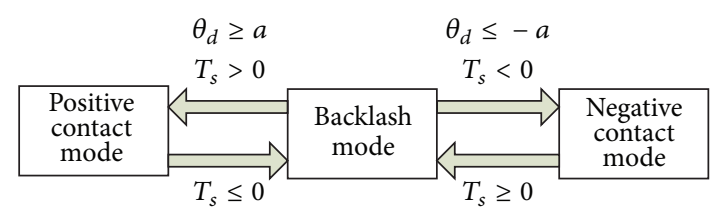

FIgURE 2: The mode transition diagram.

Dynamic equations for the two-mass system in Figure 1 are given below:

$$
\begin{aligned}
\omega_{m} & =\dot{\theta}_{m}, \\
\omega_{l} & =\dot{\theta}_{l}, \\
J_{m} \dot{\omega}_{m} & =-c_{m} \omega_{m}-T_{s}+T_{m}, \\
J_{l} \dot{\omega}_{l} & =-c_{l} \omega_{l}+T_{s},
\end{aligned}
$$

where $J_{m}$ and $J_{l}$ are the moments of inertia of the driving gear and the driven gear, respectively, $c_{m}$ and $c_{l}$ are the viscous friction coefficients of the driving gear and the driven gear, respectively, $T_{m}$ is the motor torque, $T_{s}$ is the transmitted shaft torque, $\omega_{m}$ and $\omega_{l}$ are the rotating speeds of the driving gear and the driven gear, respectively, and $\theta_{m}$ and $\theta_{l}$ are the angular displacement of the driving gear and the driven gear, respectively.

According to the dead zone model, the transmitted shaft torque between the driving gear and the driven gear is

$$
T_{s}= \begin{cases}k\left(\theta_{d}-a\right)+c \dot{\theta}_{d}, & \theta_{d}>a, \\ 0, & \left|\theta_{d}\right| \leq a, \\ k\left(\theta_{d}+a\right)+c \dot{\theta}_{d}, & \theta_{d}<-a,\end{cases}
$$

where $\theta_{d}=\theta_{m}-\theta_{l}$ is the difference of the angular displacement between the driving gear and the driven gear, $k$ and $c$ are the elastic coefficient and damping coefficient of the junction of the driving gear and the driven gear, and $2 a(\mathrm{rad})$ is the standard size of backlash.

By taking a close look at the dead zone model, we see that the contact surface of gear is assumed to be elastic. This view has also been taken in [7]. The intensity of impact caused by the collision between the driving gear and the driven gear is determined by the elastic coefficient of the junction and the size of the backlash. The bigger is the elastic coefficient of the junction, the bigger is the intensity of impact; that is, it is easier to produce larger oscillation of the transmission system. What is more, when the size of backlash is large, the oscillation of the transmission system will be also large.

\section{Controller Design}

3.1. Modes of Transmission Systems with Backlash. Transmission systems with backlash have three modes, including backlash mode, positive contact mode, and negative contact mode. Such systems switch among these modes according to a logical rule. The mode transition diagram is shown in Figure 2. 
Choose $\theta_{m}, \theta_{l}, \omega_{m}$, and $\omega_{l}$ as state variables and represent them with $x_{1}, x_{2}, x_{3}$, and $x_{4}$, respectively. So, the state equation of each mode can be obtained from (1) and (2).

State equation in the backlash mode is

$$
\left[\begin{array}{c}
\dot{x}_{1} \\
\dot{x}_{2} \\
\dot{x}_{3} \\
\dot{x}_{4}
\end{array}\right]=\left[\begin{array}{cccc}
0 & 0 & 1 & 0 \\
0 & 0 & 0 & 1 \\
0 & 0 & -\frac{c_{m}}{J_{m}} & 0 \\
0 & 0 & 0 & -\frac{c_{l}}{J_{l}}
\end{array}\right]\left[\begin{array}{c}
x_{1} \\
x_{2} \\
x_{3} \\
x_{4}
\end{array}\right]+\left[\begin{array}{c}
0 \\
0 \\
\frac{1}{J_{m}} \\
0
\end{array}\right] T_{m} .
$$

State equation in the positive contact mode is

$$
\begin{aligned}
{\left[\begin{array}{l}
\dot{x}_{1} \\
\dot{x}_{2} \\
\dot{x}_{3} \\
\dot{x}_{4}
\end{array}\right]=} & {\left[\begin{array}{cccc}
0 & 0 & 1 & 0 \\
0 & 0 & 0 & 1 \\
-\frac{k}{J_{m}} & \frac{k}{J_{m}} & -\frac{c_{m}+c}{J_{m}} & \frac{c}{J_{m}} \\
\frac{k}{J_{l}} & -\frac{k}{J_{l}} & \frac{c}{J_{l}} & -\frac{c_{l}+c}{J_{l}}
\end{array}\right]\left[\begin{array}{l}
x_{1} \\
x_{2} \\
x_{3} \\
x_{4}
\end{array}\right] } \\
& +\left[\begin{array}{c}
0 \\
0 \\
\frac{1}{J_{m}} \\
0
\end{array}\right] T_{m}+\left[\begin{array}{c}
0 \\
0 \\
\frac{k}{J_{m}} \\
-\frac{k}{J_{l}}
\end{array}\right] a .
\end{aligned}
$$

State equation in the negative contact mode is

$$
\begin{aligned}
{\left[\begin{array}{l}
\dot{x}_{1} \\
\dot{x}_{2} \\
\dot{x}_{3} \\
\dot{x}_{4}
\end{array}\right]=} & {\left[\begin{array}{cccc}
0 & 0 & 1 & 0 \\
0 & 0 & 0 & 1 \\
-\frac{k}{J_{m}} & \frac{k}{J_{m}} & -\frac{c_{m}+c}{J_{m}} & \frac{c}{J_{m}} \\
\frac{k}{J_{l}} & -\frac{k}{J_{l}} & \frac{c}{J_{l}} & -\frac{c_{l}+c}{J_{l}}
\end{array}\right]\left[\begin{array}{l}
x_{1} \\
x_{2} \\
x_{3} \\
x_{4}
\end{array}\right] } \\
& +\left[\begin{array}{c}
0 \\
0 \\
\frac{1}{J_{m}} \\
0
\end{array}\right] T_{m}+\left[\begin{array}{c}
0 \\
0 \\
-\frac{k}{J_{m}} \\
\frac{k}{J_{l}}
\end{array}\right] a .
\end{aligned}
$$

The 3 modes switch according to the rule in Figure 2. There are two kinds of switching sequences, which are described below.

(i) One switching sequence aims to switch from the negative contact mode to the positive contact mode. In that sequence, the system starts from the negative contact mode and has nonnegative transmitted shaft torque $T_{s}$. Then the system enters into the backlash mode. After a finite time (which is explained in detail in Section 3.5), the driving and driven gears contact again and the system enters into the positive contact mode, the target mode, and will stay in that mode until the next change of the reference signal occurs. (ii) The other switching sequence aims to switch from the positive contact mode to the negative contact mode. In that sequence, the system starts from the positive contact mode and switches to the backlash mode due to nonpositive $T_{s}$. Again, after a finite time, the driving and driven gears contact and the system enters into the negative contact mode and will stay there before the next change of the reference signal.

As mentioned above, the backlash mode looks like a "transient" mode. It, however, plays a critical role. When the system switches from the backlash mode to the positive or negative contact mode, the driving and driven gears contact again. If that contact is not smooth, the system suffers from an impulse and the control performance is hurt. Moreover, the time from the backlash mode to the (positive or negative) contact mode is expected to be short in order to fast-track the reference signal. In summary, we want to design a controller, particularly a controller in the backlash mode, so that the system can quickly and smoothly reach the contact mode from the backlash mode.

3.2. Controller for the Contact Mode. When the driving and driven gears contact, the transmission system becomes a linear transmission system. At this time, the difference between the system in the contact mode and the transmission system without backlash lies in that the transmitted shaft torque of the system in contact mode is smaller than that of system without backlash. Therefore, we can use the method of designing the linear controller for system without backlash to design the controller for the contact mode.

For a system without backlash, its dynamics are still governed by (1). But its transmitted shaft torque is different, which is expressed as

$$
T_{s}=k \theta_{d}+c \dot{\theta}_{d}
$$

The transfer function can be obtained from (1) and (6) as follows:

$$
\frac{\theta_{l}(s)}{T_{m}(s)}=\frac{c s+k}{d(s)},
$$

where

$$
\begin{aligned}
d(s)= & J_{m} J_{l} s^{4}+\left(c_{l} J_{m}+c J_{m}+J_{l} c_{m}+c J_{l}\right) s^{3} \\
& +\left(J_{m} k+c_{m} c_{l}+c_{m} c+c_{l} c+J_{l} k\right) s^{2}+\left(c_{m} k+c_{l} k\right) s
\end{aligned}
$$

Considering this transfer function, we can design a linear controller in the frequency domain by the Bode plot method [8]. As a result, the transmission system without backlash can track the input signal precisely through the linear controller. We can therefore implement this controller when the transmission system with backlash is in the contact mode.

3.3. Controller for the Backlash Mode. The task of the controller for the backlash mode is not only to make driving gear pass the backlash quickly, but also to minimize the 
intensity of impact when the driving gear and the driven gear contact again. So, it is required that the driving gear accelerates rapidly during the period of backlash and that the deceleration of driving gear is also large enough to minimize the relative velocity between the driving gear and the driven gear when they are close to each other. Therefore, the compensation of backlash is divided into two stages, including compensation for the period of acceleration and compensation for the period of deceleration. In order to simplify analysis, we assume that the driving gear is in the stage of positive movement, which means that the driving gear is turning to the positive contact mode from the negative contact mode or backlash mode. The analysis for negative movement is similar.

3.3.1. Controller for the Period of Acceleration. The period of acceleration means that the driving gear needs to accelerate rapidly when it just turns into backlash. The control value for the period of acceleration is

$$
T_{m}=U_{+}=-U_{1}+U_{2}+U_{3},
$$

where $U_{1}$ is the control component which is determined by the initial speed when the driving gear just turns into backlash, $U_{2}$ is the control component given by the linear controller, and $U_{3}$ is the control component determined by the current gap between the driving and driven gears.

In the period of acceleration, because the driving gear usually needs to slow down the driven gear, it accelerates in the opposite direction of its initial speed when it just turns into backlash. Therefore, the control value for the period of acceleration should contain the information of initial speed of the driving gear when it just turns into backlash. This control component containing the information of initial speed of driving gear can be set as

$$
U_{1}=k_{1}\left(\theta_{m}-\theta_{m}^{\prime}\right),
$$

where $k_{1}$ is a positive gain and $\theta_{m}$ and $\theta_{m}^{\prime}$ are the angular displacement of the driving gear which are measured by photoelectric encoders with high resolution at the moment when the driving gear enters backlash and a sampling period before it enters backlash, respectively.

The control value for the period of acceleration should also contain the information of the linear controller. The output of the linear controller contains the information of error between the angular displacement of driven gear and the given input signal. Without the information of the linear controller, the accelerated movement of the driving gear will become blind. But the linear controller is designed for the contact mode. Its control value is slightly large because the driving gear becomes no-load during the period of backlash. So, the output of the linear controller should be reduced before it is added into the control value for the period of acceleration. The control component containing the information of linear controller can be computed as

$$
U_{2}=k_{2} U_{l} \text {, }
$$

where $U_{l}$ is the output of the linear controller for the contact mode and $k_{2} \in(0,1)$ is a reduction ratio.

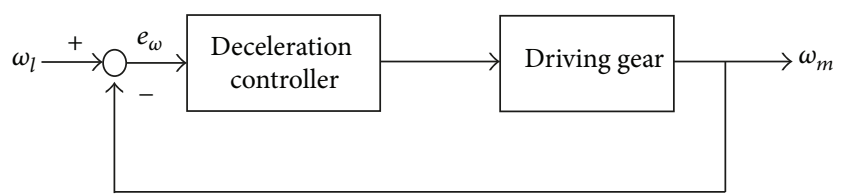

FIGURE 3: The system block diagram of the subsystem of the driving gear.

Finally, the control value for the period of acceleration should also contain the information of the current gap between the driving and driven gears, because when the driving gear turns slowly, the control value due to its initial speed becomes very small by (10) and only the control value from the linear controller affects the driving gear. In this case, it is possible that the acceleration of the driving gear is small, which, however, yields a long transition time which means poor transient performance. Therefore, besides the control component due to the initial speed and the linear controller, there also needs the control component to reflect the current gap between the driving and driven gears. This control component is proportional to the current gap. This control component plays an important role when the driving gear turns slowly and can be expressed as

$$
U_{3}=k_{3}\left(a-\theta_{d}\right),
$$

where $k_{3}$ is a positive proportional gain.

3.3.2. Controller for the Period of Deceleration. The aim of the controller in the period of deceleration is to minimize the intensity of impact when the driving gear and the driven gear contact again so that the oscillation of the transmission system can be minimized. The control value for the period of deceleration is

$$
T_{m}=U_{-}=U_{4}+U_{5},
$$

where $U_{4}$ is the control component to reduce the velocity difference between the driving and driven gears and $U_{5}$ is the control component to reduce the displacement difference between them.

For the purpose of minimizing the oscillation of the transmission system with backlash, it is expected to minimize the relative velocity between the driving and driven gears when they are close. Hence, one aim of the controller for the period of deceleration is to reduce the relative velocity between two gears during this period. For this stage, we can build a subsystem whose plant, input, and output are the driving gear, the speed of the driven gear, and the speed of the driving gear, respectively. The system block diagram is shown in Figure 3.

For such a subsystem, we use a proportional-integral controller with a dynamic proportionality coefficient to reduce the velocity error between the driving and driven gears. $U_{4}$ is set as

$$
U_{4}=k_{p 4} e_{\omega}+k_{d 4} \dot{e}_{\omega},
$$


where $k_{d 4}$ is a positive proportionality coefficient and $k_{p 4}$ is a dynamic proportionality coefficient which is related to the frequency of the input signal. The expression of $k_{p 4}$ is

$$
k_{p 4}=k_{p} * \exp \left(-\alpha * \Delta \theta_{r}\right)
$$

where $\Delta \theta_{r}$ is the difference between the input signals at two recent adjacent sampling instants, $\alpha$ is a positive proportionality coefficient which can change the attenuation rate of the exponential function, and $k_{p}$ is an appropriate proportionality coefficient in the period of deceleration when the frequency of the input signal is low. The reason for adopting such a dynamic proportional coefficient is that the proportional controller for the period of deceleration is sensitive to the change of frequency of input signals. When the frequency of the input signal is low, the velocity difference between the driving and driven gears is small, which needs a large proportion coefficient to generate an appropriate control quantity to decelerate. When the frequency of the input signal is high, the velocity difference between the driving and driven gears is large and the proportional coefficient should be decreased; otherwise, the driving gear may be reversed due to the over-large control quantity.

Beside reducing the velocity difference between the driving and driven gears, we also need to reduce the displacement difference between two gears to ensure that their relative speed is small when they contact. Therefore, there also needs a control component to reduce the displacement difference, which is chosen as

$$
U_{5}=k_{5}\left(a-\theta_{d}\right)
$$

where $k_{5}$ is a positive proportionality coefficient. It is easy to see that the above control component is related to the current gap between the driving gear and the driven gear.

\subsection{Supervisory Controller}

3.4.1. Estimation of the Current Mode. The supervisory controller judges the current mode of the transmission system by analyzing the state variables. After knowing the system's current mode, the supervisory controller switches to the corresponding controller. For the transmission system with backlash, it has three modes, including the contact mode, the backlash mode with positive movement, and the backlash mode with negative movement.

The system is in the backlash mode with positive movement when condition (17) is true:

$$
(e \geq 0) \wedge\left(\theta_{d} \leq a\right)
$$

where $\wedge$ stands for the logical and operation. The system is in the backlash mode with negative movement when condition (18) is true:

$$
(e<0) \wedge\left(\theta_{d} \geq-a\right) .
$$

The system is in the contact mode when condition (19) is true:

$$
\left((e \geq 0) \wedge\left(\theta_{d}>a\right)\right) \vee\left((e<0) \wedge\left(\theta_{d}<-a\right)\right),
$$

where $e$ is error between the output of transmission system and the input signal and $\vee$ stands for the logical or operation.

Conditions (17), (18), and (19) can be accurately verified. In order to improve the robustness of the system, we need to introduce some modifications to the above conditions. The system is determined to be in the backlash mode with positive movement when the condition in (20) is true:

$$
(e \geq 0) \wedge\left(\theta_{d} \leq a\right) \wedge\left(\left(a-\theta_{d}\right)>\beta\right) .
$$

The system is in the backlash mode with negative movement when condition (21) is true:

$$
(e<0) \wedge\left(\theta_{d} \geq-a\right) \wedge\left(\left(a+\theta_{d}\right)>\beta\right) .
$$

The system is in the contact mode when any one of the three conditions (19), (22), and (23) is true:

$$
\begin{gathered}
(e \geq 0) \wedge\left(\theta_{d} \leq a\right) \wedge\left(\left(a-\theta_{d}\right) \leq \beta\right), \\
(e<0) \wedge\left(\theta_{d} \geq-a\right) \wedge\left(\left(a+\theta_{d}\right) \leq \beta\right),
\end{gathered}
$$

where $\beta \in[0,2 a]$.

The modified conditions consider the current gap between the driving and driven gears, which means that if the gap is less than $\beta$ at the moment of the driving gear entering the backlash, the system is still treated as in the contact mode. This is because the gear often jitters slightly during the process of transmission, which means that the driving gear enters the backlash but the size of backlash is too small. If there is no threshold, the slight jitter will be judged as the backlash mode, which causes the system to switch into the controller for the backlash mode in such a small gap. The switching in this situation is not only unnecessary but may also cause larger jitter. The threshold $\beta$ will improve the robustness of the system for such a slight jitter.

3.4.2. Switching Strategy. According to the modified conditions, the supervisory controller judges the current mode of the system and switches to the corresponding controller.

(a) When the system is in the contact mode, the supervisory controller switches to the controller for the contact mode.

(b) When the system is in the backlash mode with positive movement, the supervisory controller switches to the controller for the period of acceleration first and then switches to the controller for the period of deceleration when condition (24) is true:

$$
\left(a-\theta_{d}\right)<\gamma * s
$$

where $\gamma \in(0,1)$ and $s$ is the size of the gap between the driving and driven gears at the moment when the driving gear enters into the backlash mode.

The supervisory controller switches to the linear controller from the controller for the period of deceleration when condition (25) is true:

$$
\left(\theta_{d} \geq a\right) \vee\left(\left|U_{-}\right|<\mu\right),
$$


where $\mu>0$. Note that the second part of condition (25) is to improve the robustness of the system for the change of backslash size. Because the size of backlash cannot always be equal to the standard backslash size in practice, we cannot judge whether the driving and driven gears contact only by the standard backslash size. So, the second part of the condition is necessary.

(c) When the system is in the backlash mode with negative movement, the switching strategy is similar to that in the backlash mode with positive movement. We just need to do some modifications to conditions (24) and (25) into the ones in (26) and (27), respectively.

Consider

$$
\begin{gathered}
\left(a+\theta_{d}\right)<\gamma * s \\
\left(\theta_{d} \leq-a\right) \vee\left(\left|U_{-}\right|<\mu\right) .
\end{gathered}
$$

The state information needed in the above conditions can be calculated from the angular displacement of the driving gear $\theta_{m}$ and the angular displacement of the driven gear $\theta_{l}$, which can be acquired through two photoelectric encoders with high resolution.

3.5. Stability Analysis. Most of the time, the system works in the contact modes, either the positive or negative contact mode. Therefore, we first analyze the stability of controller in the contact modes. Considering the transfer function in (7), we see that it is a traditional linear system and we can, therefore, design the controller by the Bode plot method [8]. It is easy to ensure the stability of the system in the contact modes. So, the key point of the overall stability of the system lies in the stability of the system in the backlash mode. More specifically, we will show that the aforementioned controllers can guarantee that the driving gear can go through the backlash quickly in a limited time and minimize the relative velocity between the driving and driven gears when they contact; that is, the backlash mode lasts for only a finite time.

As shown in Section 3.1, the two switching sequences work in a similar way. So, we only consider the sequence switching from the negative contact mode to the positive contact mode. When the condition in (20) is satisfied, the supervisory controller can know that the system enters into the backlash mode with positive movement and adopts the controller in (9). At that time, the driving gear leaves one side of the driven gear and accelerates to reach the other side of the driven gear. After a while, the condition in (24) is satisfied, the system enters into the period of deceleration, and the controller in (13) is taken. Due to the subcontroller $U_{4}$ in (14), the relative velocity between the driving and driven gears will start to decrease. It takes only a finite time for the driving gear to contact the driven gear because of $U_{4}$ and $U_{5}$. Note that before the driving and driven gears contact, $U_{5}$ is not zero and aims to reduce the gap between the driving and driven gears within a finite time. When the driving and driven gears contact, their relative velocity may not be zero and can cause jitter. Fortunately, their relative velocity will not get large due to the decelerating effects of $U_{4}$. When the condition in (25) is satisfied, the system leaves the backlash mode and enters

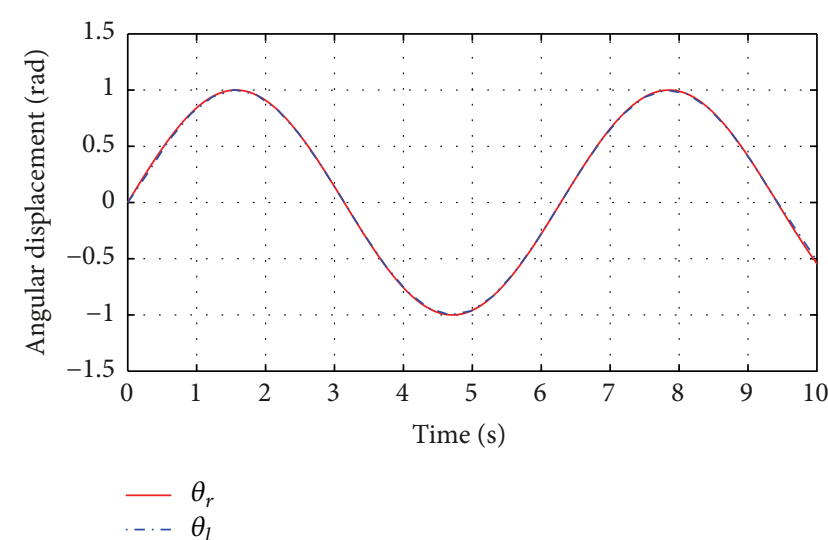

FIGURE 4: Response of the system with a sinusoidal input signal with the frequency of $0.16 \mathrm{~Hz}$.

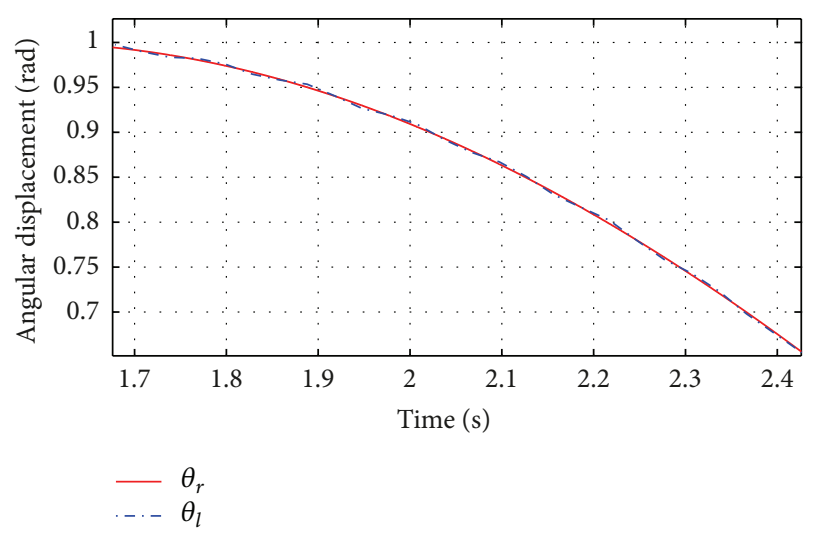

FIgURE 5: Response of the system without compensation under a sinusoidal input signal with the frequency of $0.16 \mathrm{~Hz}$.

into the positive contact mode. As discussed above, the total time to spend in the backlash mode is finite.

\section{Simulation}

According to the switching control strategy mentioned in Section 3, we use MATLAB to simulate the concerned transmission system. In the simulations, the parameters are chosen as follows:

$$
\begin{aligned}
& J_{m}=0.14, \quad c_{m}=0.3, \quad J_{l}=0.16, \quad c_{l}=0.5, \\
& k=5000, \quad c=0.5, \quad a=0.1 .
\end{aligned}
$$

The input of the system is $\theta_{r}$ and the output of the system is $\theta_{l}$.

When the input signal is a sinusoidal signal with the frequency of $0.16 \mathrm{~Hz}$, the response is shown in Figure 4. In order to see the effectiveness of the compensation methods more clearly, we will compare the response of the system without compensation and the system with compensation in Figures 5 and 6. Comparing the response in Figures 5 and 


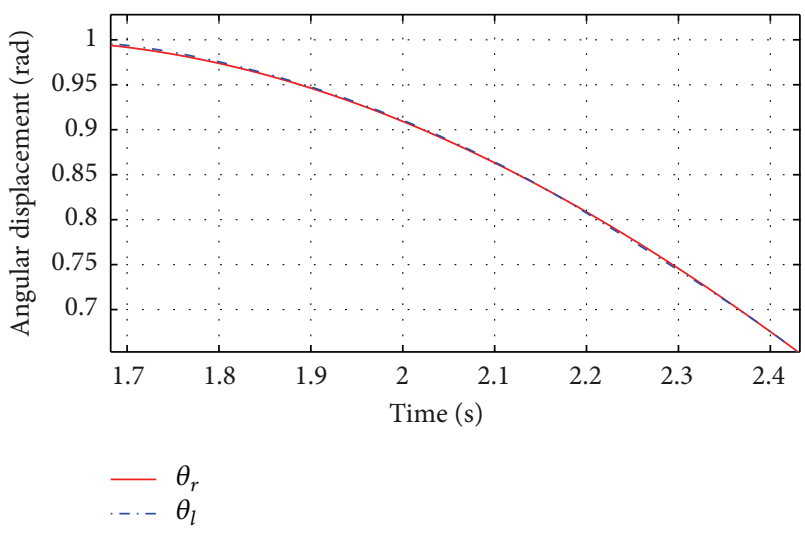

FIGURE 6: Response of the system with compensation under a sinusoidal input signal with the frequency of $0.16 \mathrm{~Hz}$.

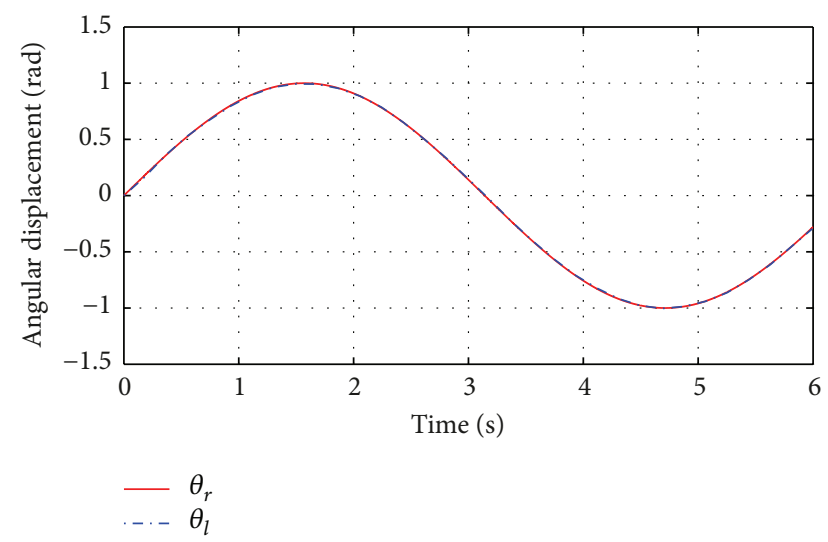

FIGURE 7: Response of the system under a sinusoidal input signal with the frequency of $0.16 \mathrm{~Hz}$ when the size of backlash is $0.08 \mathrm{rad}$.

6 , it is clear that the oscillation of the system is reduced significantly by the compensation.

Then, we will verify the effectiveness of the switching controller in the situation where the size of backlash is not the standard size of backlash. Responses of the system with the size of backlash which are $0.08 \mathrm{rad}$ and $0.12 \mathrm{rad}$ are shown in Figures 7 and 8, respectively.

We can see from Figures 4 and 5 that the system works better under the situation where the backlash size is bigger than the standard size and will generate slight jitter in the situation where the backlash size is smaller than the standard size. This is because that when the backlash size is smaller, some slight collision will occur when the driving and driven gears contact again. But the system is quite robust for the change of backlash size.

Finally, we will verify the effectiveness of the switching controller in the situation where the frequency of the input signal is varied. Responses under different frequencies of the input signal, $1 \mathrm{~Hz}, 5 \mathrm{~Hz}$, and $10 \mathrm{~Hz}$, are shown in Figures 9, 10, and 11 .

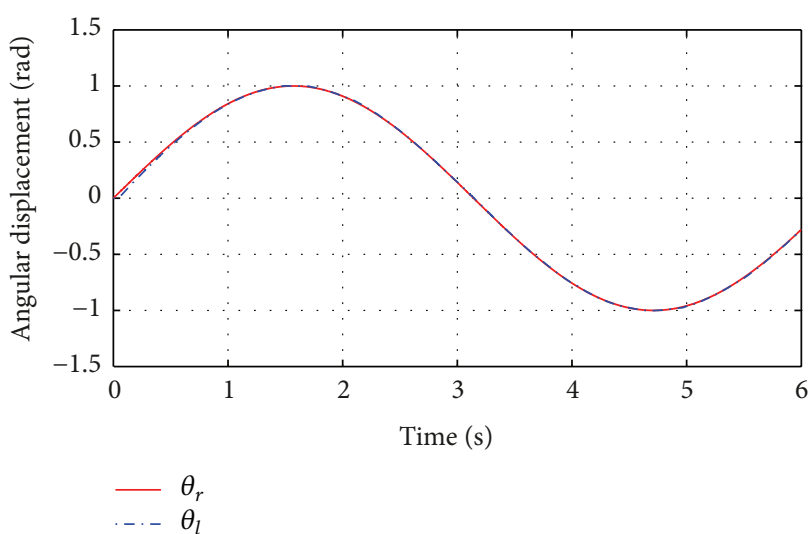

FIGURE 8: Response of the system under a sinusoidal input signal with the frequency of $0.16 \mathrm{~Hz}$ when the size of backlash is $0.12 \mathrm{rad}$.

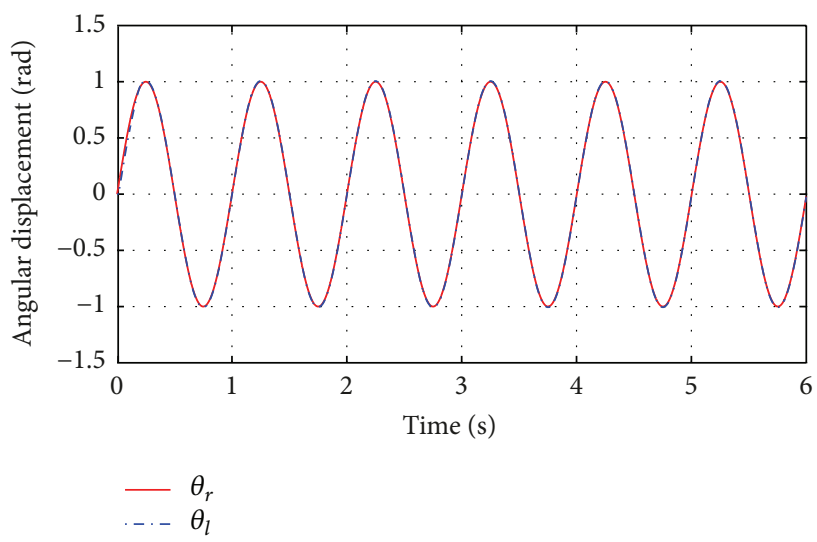

FIGURE 9: Response of the system with a sinusoidal signal with the frequency of $1 \mathrm{~Hz}$.

We see that the system still can well track the input signals when the frequency of input signal is varied. So, the system is robust for the variation of the frequency of the input signal.

\section{Conclusions}

This paper proposes a robust switching control strategy for a transmission system with unknown backlash. It analyzes the possible modes that the transmission system could lie in and designs appropriate individual controllers for each mode. In the design of the individual controllers, it considers the unknown backlash size and the variation of the frequency of the input signal. Moreover, it gives a method to estimate the current mode of the transmission system and coordinate the switching among individual controllers. Simulations are done to verify the effectiveness and robustness of the proposed switching control strategy.

\section{Conflict of Interests}

The authors declare that there is no conflict of interests regarding the publication of this paper. 


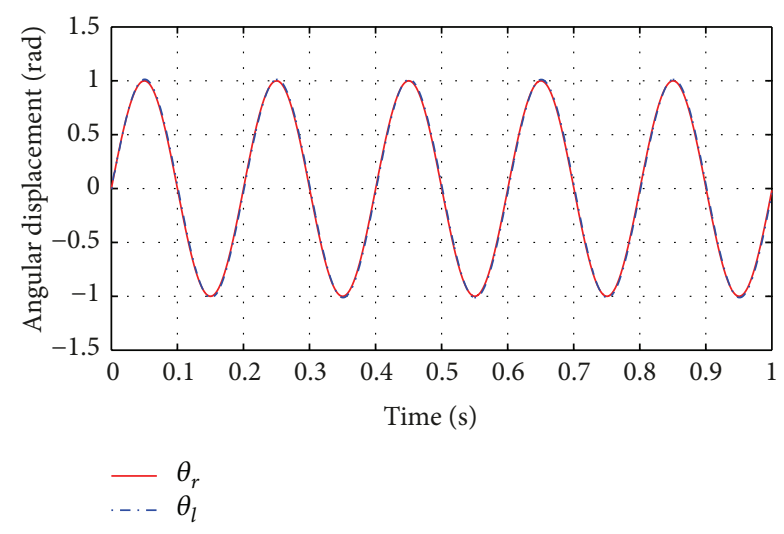

FIGURE 10: Response of the system with a sinusoidal signal with the frequency of $5 \mathrm{~Hz}$.

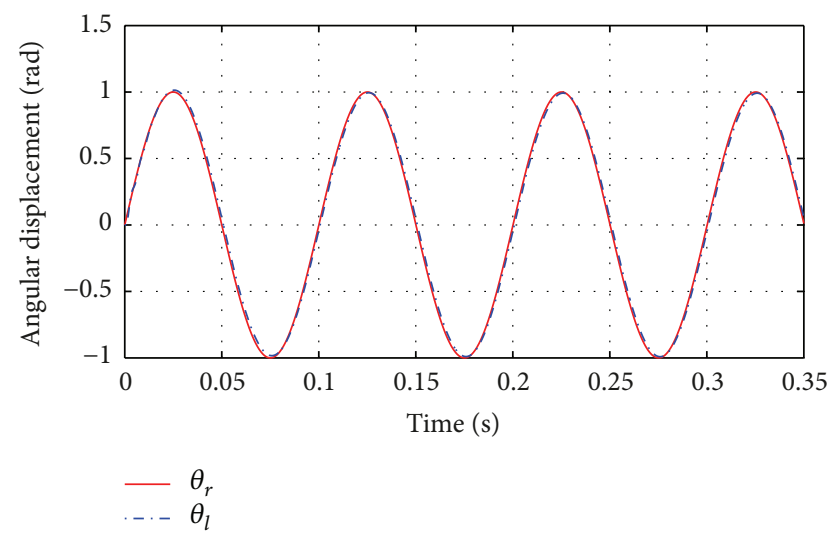

FIGURE 11: Response of the system with a sinusoidal signal with the frequency of $10 \mathrm{~Hz}$.

\section{Acknowledgments}

This work was supported in part by the National Natural Science Foundation of China (61273112) and the Open Funding from the Key Laboratory of Airborne Optical Imaging and Measurement of CAS.

\section{References}

[1] M. Nordin and P.-O. Gutman, "Controlling mechanical systems with backlash-a survey," Automatica, vol. 38, no. 10, pp. 1633$1649,2002$.

[2] W. Gawronski, J. J. Beech-Brandt, H. G. Ahlstrom Jr., and E. Maneri, "Torque-bias profile for improved tracking of the deep space network antennas," IEEE Antennas and Propagation Magazine, vol. 42, no. 6, pp. 35-45, 2000.

[3] M. B. Khan, F. M. Malik, and K. Munawar, "Switched hybrid speed control of elastic systems with backlash," in Proceedings of the IEEE International Conference on Industrial Engineering and Engineering Management (IEEM '10), pp. 1641-1644, Macao, China, December 2010.

[4] K.-H. Han, G.-O. Koh, J.-M. Sung, and B.-S. Kim, "Adaptive control approach for improving control systems with unknown backlash," in Proceedings of the 11th International Conference on
Control, Automation and Systems (ICCAS '11), pp. 1919-1923, Gyeonggi-do, Republic of Korea, October 2011.

[5] G. Tao and P. V. Kokotović, "Continuous-time adaptive control of systems with unknown backlash," IEEE Transactions on Automatic Control, vol. 40, no. 6, pp. 1083-1087, 1995.

[6] M. Grundelius and D. Angeli, "Adaptive control of systems with backlash acting on the input," in Proceedings of the 35th IEEE Conference on Decision and Control, vol. 4, pp. 4689-4694, Kobe, Japan, December 1996.

[7] J. C. Gerdes and V. Kumar, "Impact model of mechanical backlash for control system analysis," in Proceedings of the American Control Conference, vol. 5, pp. 3311-3315, Seattle, Wash, USA, June 1995.

[8] R. C. Dorf and R. H. Bishop, Modern Control Systems, Publishing House of Electronics Industry, Beijing, China, 11th edition, 2011. 


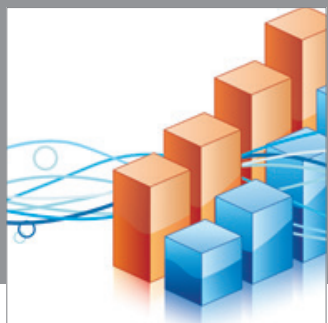

Advances in

Operations Research

mansans

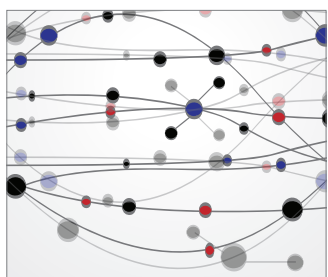

The Scientific World Journal
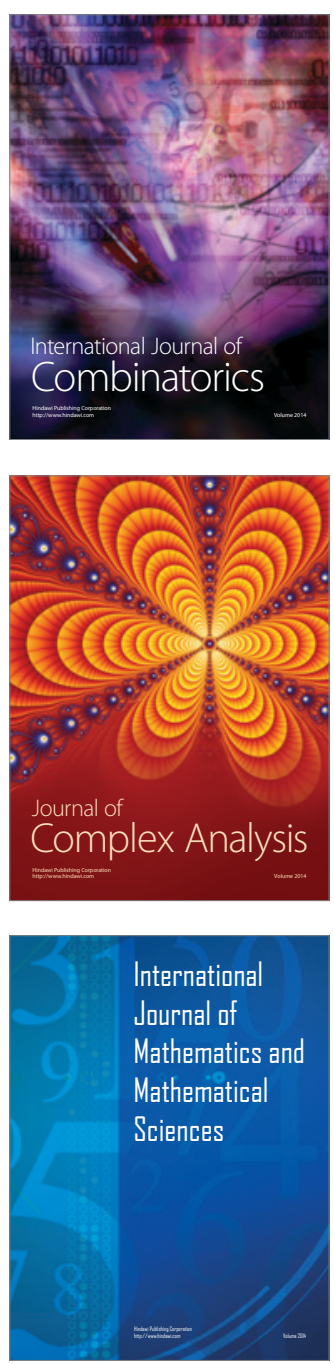
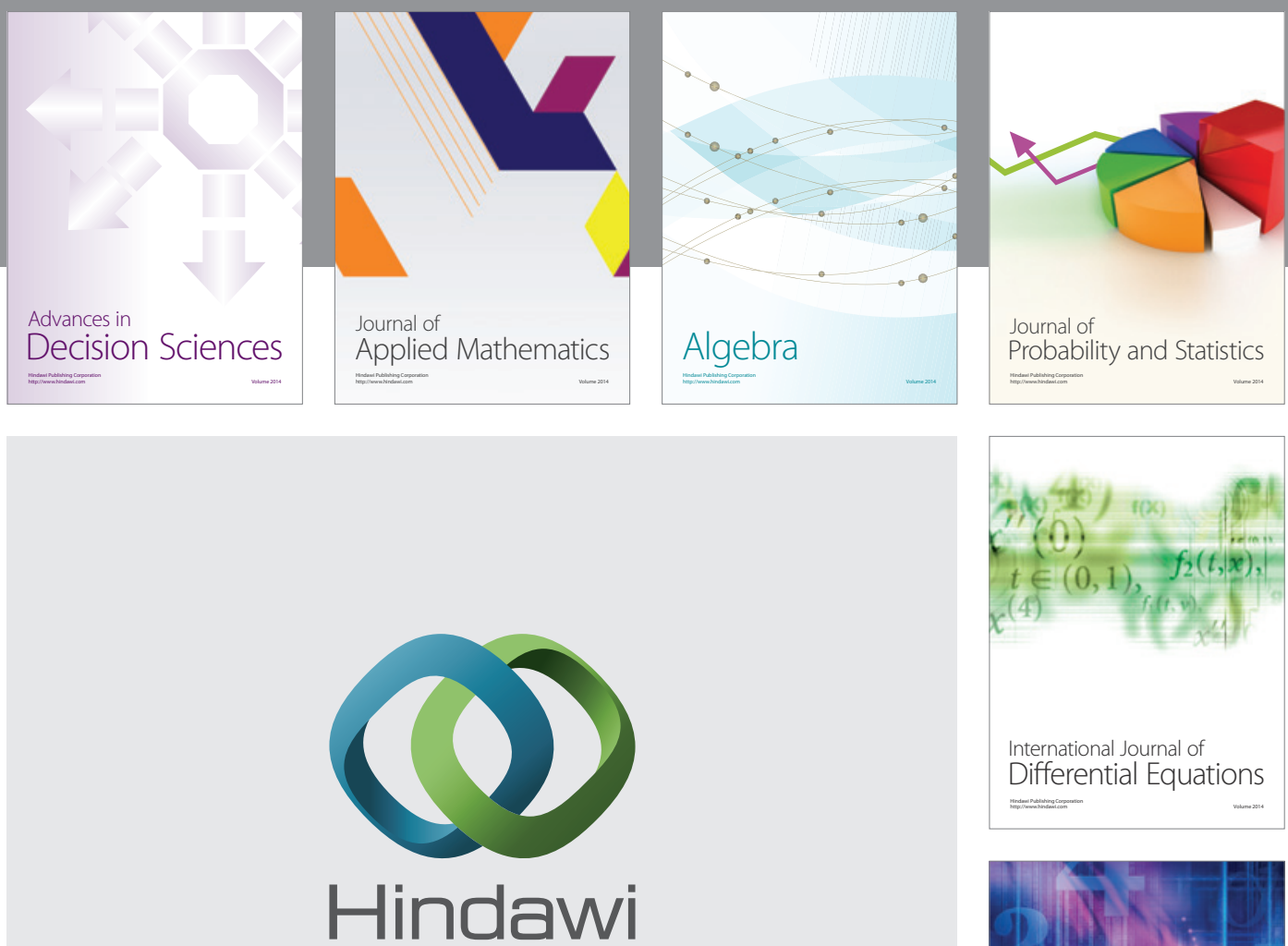

Submit your manuscripts at http://www.hindawi.com
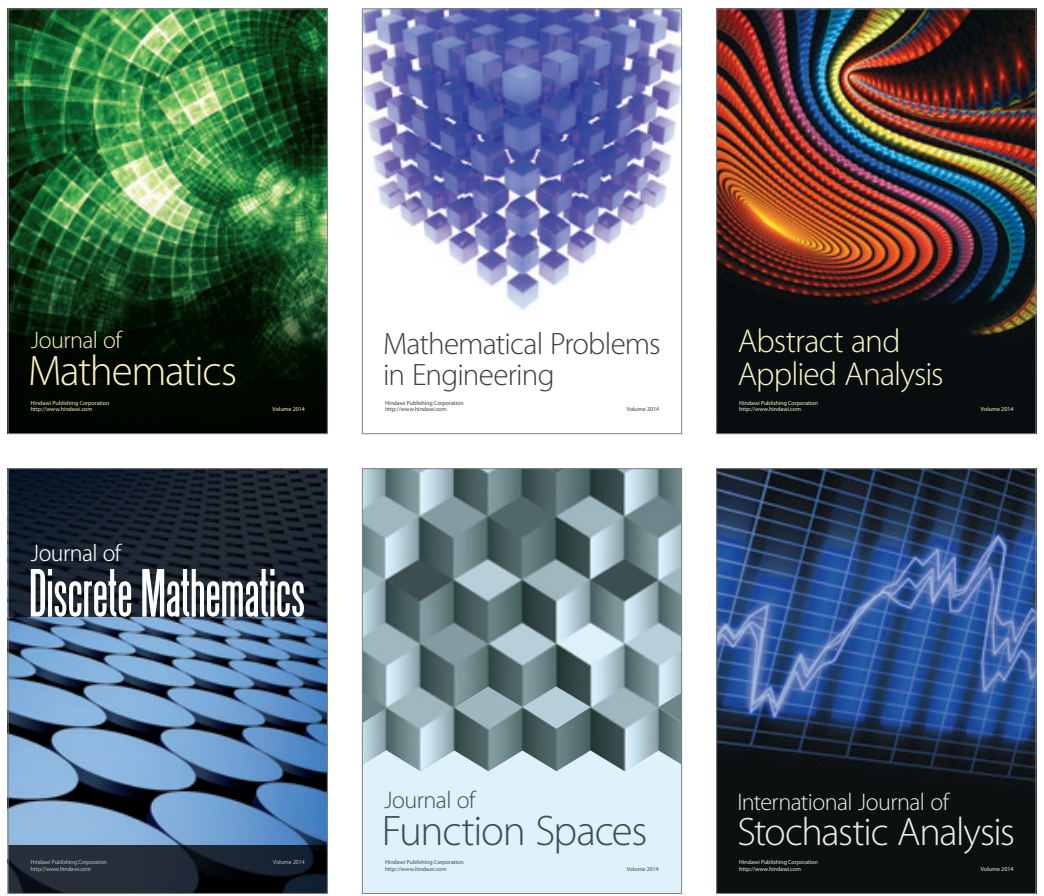

Journal of

Function Spaces

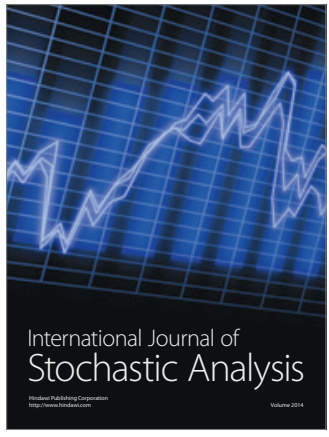

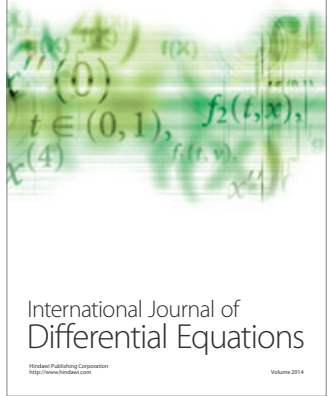
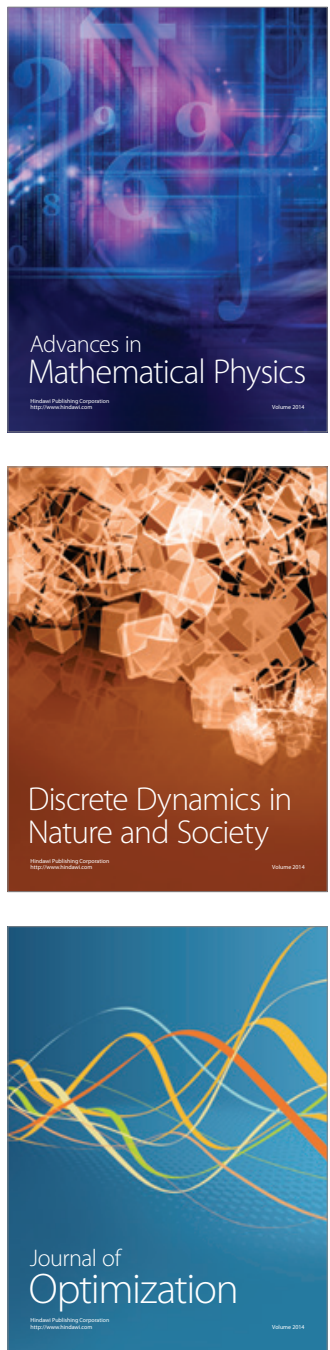